\title{
Dark matter abundance from the sequential freeze-in mechanism
}

\author{
Geneviève Bélanger, ${ }^{1}$ Cédric Delaunay $\odot,{ }^{1}$ Alexander Pukhov, ${ }^{2}$ and Bryan Zaldivar ${ }^{3}$ \\ ${ }^{1}$ Laboratoire d'Annecy-le-Vieux de Physique Théorique LAPTh, \\ CNRS-USMB, BP 110 Annecy-le-Vieux, F-74941 Annecy, France \\ ${ }^{2}$ Skobeltsyn Institute of Nuclear Physics, Moscow State University, Moscow 119992, Russia \\ ${ }^{3}$ Departamento de Fisica Teorica and Instituto de Fisica Teorica, \\ IFT-UAM/CSIC, Cantoblanco, 28049, Madrid, Spain
}

(Received 20 May 2020; accepted 24 July 2020; published 14 August 2020)

\begin{abstract}
We present a thorough analysis of the sequential freeze-in mechanism for dark matter production in the early Universe. In this mechanism the dark matter relic density results from pair annihilation of mediator particles which are themselves produced by thermal collisions of standard model particles. Below some critical value of the mediator coupling to standard model fields, this sequential channel dominates over the usual freeze-in where dark matter is directly produced from thermal collisions, even when the mediator is not in thermal equilibrium. The latter case requires computing the full nonthermal distribution of the mediators, for which finite temperature corrections are particularly important.
\end{abstract}

DOI: $10.1103 /$ PhysRevD.102.035017

\section{INTRODUCTION}

The nature of the dark matter $(\mathrm{DM})$ is perhaps the most acute open question in particle physics. Despite the strong observational evidence for an important DM component in the Universe, most of its properties remain unknown. Requiring that the DM be a thermal relic implies nongravitational interactions with ordinary matter. This nourishes hope to probe the DM in our local environment, either by detecting DM particles, directly in underground experiments or indirectly through the products of DM annihilation within our galactic neighborhood, or by producing them at colliders.

In particular, the hypothesis of a new DM particle around the electroweak scale has been tested extensively and the lack of evidence for DM from these searches triggered a growing interest in exploring a wider class of DM models. One possibility is that the $\mathrm{DM}$ and/or the mediator connecting it to the standard model (SM) is below the GeV scale, thus leaving no traces in nuclear-scattering based direct detection experiments [1] and colliders. This regime can be tested nonetheless with different experimental probes [2-7]. Besides, a mediator in the sub-GeV mass range also helps in resolving small-scale problems related to DM [8-10].

Published by the American Physical Society under the terms of the Creative Commons Attribution 4.0 International license. Further distribution of this work must maintain attribution to the author(s) and the published article's title, journal citation, and DOI. Funded by SCOAP ${ }^{3}$.
Another possibility is that the DM particle still lies above the $\mathrm{GeV}$ scale but signals in standard searches are suppressed because it interacts only very weakly with the SM. In this scenario, the DM is never in equilibrium with the SM in the early Universe and is instead produced by freezein through pair annihilation or decay of particles in the thermal bath $[11,12]$. The DM is generally assumed to be singlet under the SM group and part of a hidden sector that couples to the SM through renormalizable portal operators or with a mediator particle. Despite its tiny couplings with the SM fields the DM relic density often remains detectable in existing experiments [13], like direct detection when the mediator is light $[14,15]$ or indirect detection for decaying DM [16,17]. Frozen-in DM could also be tested in cosmology [18-20] and at colliders through signatures involving displaced vertices and/or long-lived particles [21-27] including with detectors located some distance from the interaction point [28-31].

In this work we consider scenarios where dark sector particles are feebly coupled to the SM and where the DM relic is produced nonthermally through the freeze-in mechanism. We assume the mediator mass is small, at the $10 \mathrm{MeV}$ scale, and is a scalar, for simplicity. ${ }^{1}$ Lighter mediators also have interesting phenomenology but suffer severe constraints from Big Bang nucleosynthesis. The light mediator can potentially provide an explanation for anomalies observed at the cluster scale [34,35] and enhances the DM-nucleus scattering cross section, which offers the possibility to probe this scenario in direct detection. We will

${ }^{1}$ The case of a light vector is qualitatively similar; see e.g., Refs. [32,33]. 
consider a simplified model with a hadrophilic scalar mediator which couples only to light quarks, thus alleviating several constraints that affect Higgs portal models where couplings to heavy quarks dominate [36-38]. This framework will be sufficient to illustrate the main phenomenological properties that are expected to be relevant for a larger set of models.

For freeze-in to take place, the product of mediator couplings to the SM and to DM must be very small, $\mathcal{O}\left(10^{-12}-10^{-10}\right)$, while their relative size remains a free parameter [12]. In models where DM is much heavier than the mediator, DM can generically be produced via pair annihilation of SM particles or mediators, assuming the latter are in equilibrium with the SM thermal bath. Here, we point out that even when the mediator coupling to the SM is too small for the mediator to ever reach equilibrium, a finite density of mediators can be produced through SM induced processes. The contribution of such nonthermal mediators to DM production parametrically dominates over that of pair annihilation of SM particles. This new phase of DM production, called sequential freeze-in [39,40], requires first solving for the momentum distribution of the mediator yield before using it for DM production. For this we solved the unintegrated Boltzmann equation rather than assuming $\phi$ to be in kinetic equilibrium with the thermal bath. Because the mediator is much lighter than DM, the tail of the mediator distribution is most relevant for DM production. Moreover we show that thermal effects, which we approximate by taking into account thermal masses for quarks, gluons and photons ${ }^{2}$ play an important role in mediator production. Finally, our calculation takes into account the Bose-Einstein and Fermi-Dirac distributions for bosons and fermions, respectively, rather than simply using Maxwell-Boltzmann (MB) distributions. We show that these effects lead to large corrections in the calculation of the relic density [42].

The paper is organized as follows. We first present the simplified model and discuss at length the possible DM production mechanisms, giving an extensive description of the specific case where the mediator is not in thermal equilibrium (Sec. III). With the complete calculation of DM production in hand (Sec. IV), we then determine the potential of current and future direct detection (DD) experiments to probe this model (Sec. V) and examine numerous astrophysics and terrestrial constraints (Sec. VI). Our conclusions are presented in Sec. VII. The Appendixes contain details on the reaction rates for mediator production as well as an approximate analytical solution for the mediator distribution.

\section{SIMPLIFIED DARK MATTER MODEL}

Consider a simplified model for the dark sector which comprises a Dirac fermion $\chi$ (the DM candidate) interacting

\footnotetext{
${ }^{2}$ A recent study showed the importance of the thermal mass for DM production from thermal photon decays [41].
}

with SM quarks through a real scalar mediator $\phi$ with parity-preserving renormalizable couplings

$$
-\mathcal{L}_{\text {int }}=y_{\chi} \phi \bar{\chi} \chi+y_{q} \phi \bar{q} q
$$

where the Lagrangian above is written below the scale of electroweak (EW) symmetry breaking. Both dark states are neutral under the SM gauge group and $\chi$ is assumed odd under a $\mathbb{Z}_{2}$ symmetry and is thus stable. In the following, we will only consider a nonzero coupling for the firstgeneration up quark, $q=u$. $^{3}$

The question of the origin of the interactions in Eq. (1) might be raised. If $\phi$ is a SM singlet, we envisage two simple possibilities. For instance, $\phi$ and the SM could connect directly through the (renormalizable) Higgs portal, giving $y_{f}=m_{f} / v \times \sin \theta$ where $v \approx 246$ is the SM Higgs vacuum expectation value breaking EW symmetry and $\theta$ is a mixing angle. In this case though, the mediator would couple to all charged fermions and its interactions with the third family would dominate. ${ }^{4}$ In the absence of Higgs portal, $\phi$ could couple to SM fermions through interactions that involve additional states beyond the SM. For example, SM quarks could mix with new vectorlike fermions that undergo Yukawa interactions with $\phi$. In the limit that these additional states are heavy, characterized by a mass scale $\Lambda$, their dynamics will be captured by nonrenormalizable operators like $\left(x_{f} / \Lambda\right) \bar{Q} H f_{R} \phi+$ H.c., with $Q$ and $f=u$, $d$ denoting, respectively, $\mathrm{SU}(2)_{L}$ doublets and singlets. In this case $y_{f} \sim x_{f} v / \Lambda$ and coupling to the first family could dominate depending on the UV dynamics setting the flavor structure of the $x_{f}$ couplings. Moreover, taking $\Lambda \gg v$ would provide a simple rationale for the feeble couplings characterizing the freeze-in mechanism which we ought to consider in this article.

We further assume that the UV completion does not induce possible $\phi^{3}$ and $\phi^{4}$ self-interactions, as well as Higgs portal interaction $\phi^{2} H^{\dagger} H$, where $H$ is the SM Higgs doublet. Since these operators and the Lagrangian in Eq. (1) share the same symmetry, nonzero couplings are nevertheless generated by loops of low-energy degrees of freedom. Such loops are UV sensitive, yet naive dimensional analysis [46] yields useful order-of-magnitude estimates, namely $\lambda_{3} \sim y_{\chi}^{3} / 16 \pi^{2}, \lambda_{4} \sim y_{\chi}^{4} / 16 \pi^{2}$ and $\lambda_{\phi H} \sim y_{q}^{2}\left(m_{q} / v\right)^{2} / 16 \pi^{2}$, respectively. Mediator particles may be produced by Higgs boson annihilation through the Higgs portal, while self-interactions, in addition, would affect the energy distribution of mediator particles produced by freeze-in, eventually bringing them to reach a dark thermal equilibrium [47]. Assuming the above estimates of the loop-induced couplings, we find however that the rates for energy transfer

\footnotetext{
${ }^{3}$ A similar model was considered in [43] and flavor issues in this class of models were addressed in $[44,45]$.

${ }^{4}$ See Ref. [38] for a detailed study of thermal DM production in this scenario.
} 
within the dark sector and particle creation from the Higgs portal are much slower than Hubble and thus negligible for the range of $y_{\chi}$ and $y_{q}$ couplings considered.

At energies below $\Lambda_{\mathrm{QCD}} \approx 200 \mathrm{MeV}$, quarks are no longer free and the $\phi$ interactions with the SM are better described in terms of hadronic resonances. We will limit ourselves here to protons, neutrons and pions. The lowenergy interaction Lagrangian is

$$
-\mathcal{L}_{\text {int }}^{\text {had }}=\sum_{N=p, n} y_{N} \phi \bar{N} N+y_{\pi} \phi \pi \pi,
$$

where the hadronic couplings are related to the fundamental quark coupling by matching. For first generation quarks, the coupling to nucleons is

$$
y_{N}=y_{q} \frac{m_{N}}{m_{q}} f_{q}^{N},
$$

where the $f_{q}^{N}$,s are nuclear form factors whose values are extracted from matching nuclear data with lattice simulations $[48,49]$. For energies well below $4 \pi f_{\pi}$, where $f_{\pi} \approx$ $93 \mathrm{MeV}$ is the pion decay constant, the coupling to pions can be derived from chiral perturbation theory, which gives

$$
y_{\pi}=y_{q} \frac{m_{\pi}^{2}}{m_{u}+m_{d}},
$$

at leading order for first generation quarks [50,51].

Here the mediator is $\mathbb{Z}_{2}$-even and thus unstable. For $m_{\phi}<2 m_{\pi}$ the leading decay channel is in two photons through loops of charged hadrons.

$$
\Gamma_{\phi \rightarrow \gamma \gamma}=\frac{\alpha^{2} m_{\phi}^{3}}{144 \pi^{3}}\left|\frac{y_{N}}{m_{N}}+\frac{y_{\pi}}{8 m_{\pi}^{2}}\right|^{2},
$$

which corresponds to a lifetime of $\tau_{\phi} \approx 8.4 \times 10^{-14} \mathrm{sec} / y_{q}^{2}$ for $m_{\phi}=10 \mathrm{MeV}$ and a $q=u$ coupling evaluated at the matching scale $\mu=2 \mathrm{GeV}$.

In order to retain the possibility of velocity-dependent cross sections for DM self-interactions required by clusters anomaly, we will consider the light mediator limit $m_{\phi} \ll m_{\chi}$ with $m_{\phi}>1 \mathrm{MeV}$. For mediators below the $\mathrm{MeV}$ scale, very strong constraints apply on scalar couplings to nucleons which basically excludes freeze-in production of DM [33].

A light mediator is typically in tension with big bang nucleosynthesis (BBN), unless it decouples and decays at earlier times. A mediator still coupled to the thermal bath when BBN starts would hasten Hubble expansion relative to the SM and in turn alter the abundance of light elements beyond an acceptable level. This problem is easily avoided by considering mediators with mass of few $\mathrm{MeV}$ or larger, which would decouple when becoming nonrelativistic. Even if the mediator decouples before BBN, its late decay into photons would reduce the baryon-to-photon ratio, whose variation is bounded between $\mathrm{BBN}$ and $\mathrm{CMB}$ eras [52]. This strong constraint can be evaded by introducing in $\mathcal{L}_{\text {int }}$ an additional interaction of the mediator to, for instance, neutrinos, such as $-y_{\nu} \phi \bar{\nu} \nu$. In that case, the decay width into neutrinos $\Gamma_{\phi \rightarrow \nu \bar{\nu}}=y_{\nu}^{2} m_{\phi} / 8 \pi$ can be sufficiently large to clean out the Universe of mediators before BBN starts with a relatively small coupling of $y_{\nu} \sim \mathcal{O}\left(10^{-10}\right)$ which has no significant impact on the DM phenomenology. Note that the mediator has to decay into neutrinos sufficiently early so that most of them thermalize before neutrino decoupling at $T \approx$ few $\mathrm{MeV}$.

In order to avoid cosmological constraints while maximizing the effect of the light mediator on DM phenomenology we conservatively set $m_{\phi}=10 \mathrm{MeV}$ in the remainder of this article. Moreover, we focus on DM in the $1-100 \mathrm{GeV}$ mass range where significant DM-nucleus scattering signals in next-generation direct detection experiments are expected.

\section{MEDIATOR FREEZE-IN PRODUCTION}

The mediator contribution to DM production $\gamma_{\phi \phi \rightarrow x \bar{x}}$ requires knowledge of the phase-space distribution of $\phi$ particles, $f_{\phi}(p, T)$. The latter is obtained from solving the (unintegrated) Boltzmann equation

$$
E\left(\partial_{t}-H p \partial_{p}\right) f_{\phi}=C\left[f_{\phi}\right],
$$

where $p$ and $E=\left(m_{\phi}^{2}+p^{2}\right)^{1 / 2}$ are, respectively, the 3 -momentum and energy of $\phi$ in the frame of the thermal bath, $H$ is the Hubble rate and $C\left[f_{\phi}\right]$ is the collision term. Solving Eq. (6) is numerically challenging partly because of the $\partial_{p}$ term which accounts for the momentum change due to Hubble expansion. It is however possible to factor out this effect by introducing the dimensionless variable

$$
q \equiv \frac{p}{T_{0}}\left[\frac{s\left(T_{0}\right)}{s(T)}\right]^{1 / 3}=\frac{p}{T}\left[\frac{h_{\mathrm{eff}}\left(T_{0}\right)}{h_{\mathrm{eff}}(T)}\right]^{1 / 3},
$$

where $h_{\mathrm{eff}}(T)$ is the number of degrees of freedom contributing to the entropy density $s(T)=2 \pi^{2} / 45 h_{\text {eff }} T^{3}$ and $T_{0} \approx 2.3 \times 10^{-4} \mathrm{eV}$ is the photon temperature today. The entropy ratio in Eq. (7) further accounts for the slowdown of the Hubble rate due to the decoupling of species across the cosmic history. For $T \gtrsim \mathcal{O}(1 \mathrm{GeV})$, $q \approx 0.4 \times p / T$.

In terms of this reduced momentum variable Eq. (6) is brought to a one-derivative differential equation

$$
x \bar{H} \partial_{x} f_{\phi}(q, x)=E^{-1} C\left[f_{\phi}\right],
$$

here expressed in terms of $x=m_{\chi} / T$, with $\bar{H} \equiv H$ / $\left[1+1 / 3 \times d \log h_{\text {eff }} / d \log T\right]$. This equation can be solved for fixed $q$. 
The other complication lies in the form of the collision term $C\left[f_{\phi}\right]$. Several interactions contribute to bring the mediator in thermal contact with the SM bath. The dominant contribution arises from QCD processes with a single $\phi$ in the final state. Those are $g q \rightarrow q \phi$ and $\bar{q} q \rightarrow g \phi$ as well as $g \rightarrow q \bar{q} \phi$ once thermal corrections are included (see Sec. III B). We also include subdominant electromagnetic processes $q \gamma \rightarrow q \phi$ and $q \bar{q} \rightarrow \gamma \phi$ which contribute at the $\mathcal{O}(10 \%)$ level. Pair production of $\phi$ is suppressed by a factor of $\mathcal{O}\left(4 \pi y_{q}^{2} / \alpha_{s}\right)$, where $\alpha_{s}$ is the QCD coupling, and is therefore negligible in the limit $y_{q} \ll 1$ that is required for freeze-in. Hence, we have

$$
E^{-1} C\left[f_{\phi}\right]=\hat{\gamma}_{q \bar{q} \leftrightarrow V \phi}+2 \hat{\gamma}_{q V \leftrightarrow q \phi}+\hat{\gamma}_{g \leftrightarrow q \bar{q} \phi},
$$

where $\hat{\gamma}_{i} \equiv E^{-1} C_{i}\left[f_{\phi}\right]$ is the collision term associated with the process $i$. A sum over $V=g, \gamma$ is implicit in Eq. (9) and the factor 2 multiplying the second term on the right-hand side accounts for the charge-conjugated process $\bar{q} V \leftrightarrow \bar{q} \phi$. Note that the last term is only sourced by thermal plasma effects.

\section{A. Unintegrated collision rates}

The collision terms for $2 \rightarrow 2$ and $1 \rightarrow 3$ processes are generally expressed as (upper/lower sign applies to fermions/bosons)

$$
\begin{aligned}
\hat{\gamma}_{\text {in } \leftrightarrow \text { out }+\phi}= & \frac{1}{2 E} \int \prod_{i} \frac{d^{3} p_{i}}{(2 \pi)^{3} 2 E_{i}}(2 \pi)^{4} \delta^{(4)}\left(P_{\text {in }}-P_{\text {out }}-p\right) \\
& \times\left[f_{\text {in }}\left(1 \mp f_{\text {out }}\right)\left(1+f_{\phi}\right)\left|\mathcal{M}_{\text {in } \rightarrow \text { out }+\phi}\right|^{2}\right. \\
& -(\text { in } \leftrightarrow \text { out }+\phi)],
\end{aligned}
$$

where $\mathcal{M}$ denotes the scattering amplitude with initial and final state spins and colors summed over, the in (out) label denotes all the incoming (outgoing) particles other than $\phi$ with total momentum $P_{\text {in(out) }}$, the index $i$ runs over bath particles and $f_{\text {in }}\left(1 \mp f_{\text {out }}\right)=f_{1} f_{2}\left(1 \mp f_{3}\right)$ for $2 \rightarrow 2$ scattering and $f_{1}\left(1 \mp f_{2}\right)\left(1 \mp f_{3}\right)$ for $1 \rightarrow 3$ decay processes. The first term inside the bracket represents the creation contribution from the process in $\rightarrow$ out $+\phi$, while the second one accounts for depletion from its reverse counterpart out $+\phi \rightarrow$ in.

These two contributions, respectively denoted by $\hat{\gamma}_{\text {in } \rightarrow \text { out }+\phi}$ and $\hat{\gamma}_{\text {out }+\phi \rightarrow \text { in }}$, are related thanks to equilibrium conditions. Indeed, since particles 1, 2 and 3 are in thermal equilibrium with distribution $f_{i}=\left[\exp \left(E_{i} / T\right) \pm 1\right]^{-1}$, energy conservation, $E_{\text {in }}=E_{\text {out }}+E$, implies

$$
f_{\text {out }}\left(1 \mp f_{\text {in }}\right)=e^{E / T} f_{\text {in }}\left(1 \mp f_{\text {out }}\right),
$$

where $E$ is the energy of $\phi$ in the rest frame of the plasma. Moreover, in the absence of $C P$ violation (as in the simplified model of interest) $\mathcal{M}_{\text {in } \rightarrow \text { out }+\phi}=\mathcal{M}_{\text {out }+\phi \rightarrow \text { in }}$, hence we have

$$
\hat{\gamma}_{\text {out }+\phi \rightarrow \text { in }}=\frac{e^{E / T} f_{\phi}}{1+f_{\phi}} \hat{\gamma}_{\text {in } \rightarrow \text { out }+\phi} .
$$

Then, the generic expression for the collision terms in Eq. (8) simplifies to

$$
\hat{\gamma}_{\text {in out }+\phi}=R(x, q)\left[\left(1+f_{\phi}\right) e^{-E / T}-f_{\phi}\right],
$$

where

$$
R(x, q) \equiv f_{\phi}^{-1} \hat{\gamma}_{\text {out }+\phi \rightarrow \text { in }}=\frac{e^{E / T}}{1+f_{\phi}} \hat{\gamma}_{\text {in } \rightarrow \text { out }+\phi}
$$

is the rate of the reaction in $\leftrightarrow$ out $+\phi$. Note that rates can be evaluated considering either the creation or depletion process of $\phi$, thanks to equilibrium of SM particles. Finally, in the limit where this rate is much faster than the Hubble rate, $R / \bar{H} \gg 1$, the bracket in Eq. (13) goes to zero, meaning that $\phi$ reaches thermal equilibrium with the $\mathrm{SM}, f_{\phi} \rightarrow\left(e^{E / T}-1\right)^{-1}$.

For $2 \rightarrow 2$ scattering, it is more convenient to consider depletion processes (with initial state $\phi$ ) to calculate the associated rates. Indeed, in this case, neglecting Pauli blocking and stimulated emission effects, ie. taking $\left(1 \mp f_{1}\right)\left(1 \mp f_{2}\right) \simeq 1, \hat{\gamma}_{3 \phi \rightarrow 12}$ admits a simple expression in terms of the scattering cross section $\sigma_{3 \phi \rightarrow 12}$, and

$$
R_{\text {scat }} \simeq \frac{g_{3}}{(2 \pi)^{3}} \int d^{3} p_{3} f_{3} \sigma_{3 \phi \rightarrow 12} v_{\mathrm{M} ø 1},
$$

where $v_{\mathrm{M} \varnothing \mathrm{l}}$ is the Møller velocity and $g_{i}$ is the number of degrees of freedom of particle $i$.

On the other hand, considering creation processes (with final state $\phi$ ) is more convenient for decay. Within the same approximation, the collision term for the $1 \rightarrow 3$ process is expressed in terms of the differential (partial) decay width $d \Gamma_{1 \rightarrow 23 \phi} / d^{3} p$ in the frame of the thermal bath, yielding

$$
R_{\text {decay }} \simeq g_{1} e^{E / T} \int d^{3} p_{1} f_{1} \frac{d \Gamma_{1 \rightarrow 23 \phi}}{d^{3} p} .
$$

We refer the interested reader to Appendix A for a fully detailed evaluation of the integrals in Eqs. (15) and (16). Note that integrating $\hat{\gamma}$ over the $\phi$ phase space yields $\int d^{3} p /(2 \pi)^{3} \hat{\gamma}_{3 \phi \rightarrow 12}=\left\langle\sigma_{3 \phi \rightarrow 12} v\right\rangle n_{3} n_{\phi}$ and $\int d^{3} p /(2 \pi)^{3} \hat{\gamma}_{1 \rightarrow 23 \phi}=\left\langle\Gamma_{1 \rightarrow 23 \phi}\right\rangle n_{1}$, where $\langle\cdots\rangle$ denotes thermal averaging and $n$ the number density.

The reaction rates depend on temperature and rescaled momentum $q$, and they are typically faster for low momenta. For instance, as we show in Appendix A, the rate of $2 \rightarrow 2$ reactions approximately scales like $1 / q$ at large $q$. As a result, energetic $\phi$ particles, whose momentum is larger than temperature, take more time to reach equilibrium relative to less energetic ones. For sake of illustration the rates of all 


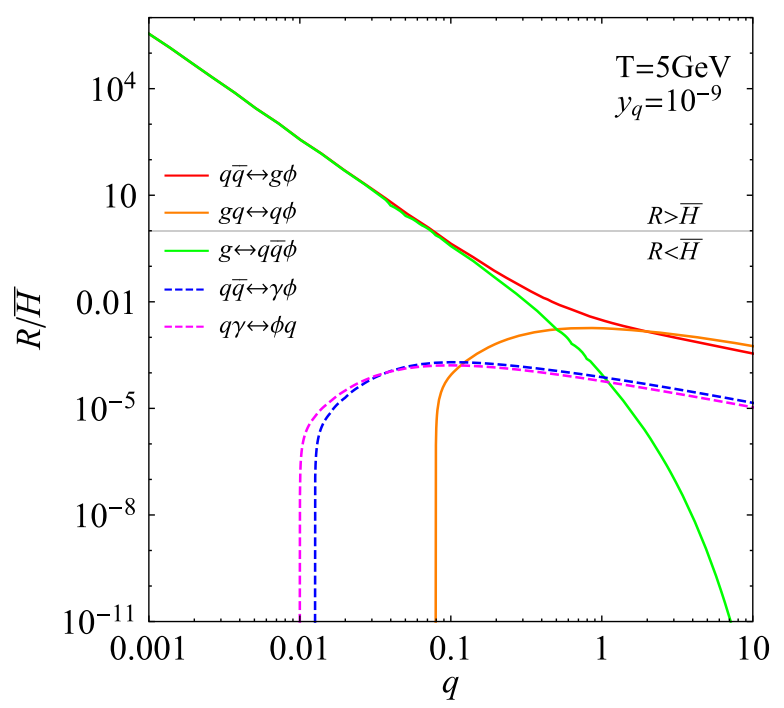

FIG. 1. Reaction rates relative to the Hubble rate for all processes relevant in $\phi$ production, including thermal masses for SM particles.

relevant processes, including important plasma effects that we discuss in the next section, are shown in Fig. 1 for $T=5 \mathrm{GeV}$. Rates for different temperatures show similar behavior (see Appendix A).

\section{B. Finite temperature corrections}

Thermal corrections to the collision term $C\left[f_{\phi}\right]$ are implemented as follows. The perturbative formulation of gauge theory in vacuum (in powers of gauge coupling) breaks down in the presence of a hot medium due to the emergence of an external scale, the temperature $T$ of the plasma. Gauge theory at finite temperature can still be formulated consistently only with a reorganized perturbative series where a certain class of diagrams needs to be resummed [53,54] (see also Ref. [55] for a recent review). We limit ourselves here to the so-called hard-thermal-loop (HTL) approximation [56] which only resums the higherorder loop diagrams associated with soft momenta $\sim g T \lesssim$ $T$ where $g$ denotes gauge couplings. In this approximation, fermions and gauge bosons are quasiparticles with temperature dependent masses.

Gauge bosons at finite temperature have polarizationdependent dispersion relations [57]. However, the propagators of transverse and longitudinal polarizations have the same pole at zero momentum, which is given by the plasma frequency related to the Debye screening of the (chromo)electric field in a medium, and only develop small differences for nonzero momentum. We neglect these differences here and in the calculation of scattering amplitudes we only replace the gauge propagator by a massive one with a pole mass given by the thermal Debye mass. To leading order in gauge coupling those are [58]

$$
m_{g}^{2}(T)=g_{s}^{2} T^{2} / 3\left[N_{c}+n_{f}(T) / 2\right],
$$

for gluons and

$$
m_{\gamma}^{2}(T)=e^{2} T^{2} n_{\mathrm{ch}}(T) / 3,
$$

for photons, where $n_{f}(T)$ and $n_{\mathrm{ch}}(T)$ are the number of active (namely satisfying $m \lesssim T$ ) quark flavors and charged particles in the plasma, respectively.

Quark dispersion relations are also modified at finite temperature with the emergence of hole excitations [59]. Nevertheless, particle and hole states are together well described by a quark propagator with a momentum independent thermal mass [60]. We therefore neglect these differences and simply add to the quark propagator the thermal mass [61],

$$
m_{q}^{2}(T)=g_{s}^{2} T^{2} / 6+e^{2} T^{2} Q_{q}^{2} / 8,
$$

where $Q_{q}$ is the quark electric charge, in the calculation of scattering amplitudes.

Finally, interaction vertices also receive finite temperature corrections. Those are captured to a very good approximation by renormalizing all coupling constants at the scale of the first Matsubara mode, $\mu_{R}=2 \pi T$, using renormalization group equations in vacuum [60].

There are several important implications of the plasma effects described above on mediator production. First of all, the quark thermal mass of $\mathcal{O}\left(g_{s} T\right)$ regulates the forwardenhancement of $t$-channel diagrams and thus strongly suppresses the production cross section compared to the zero-temperature limit. This is particularly visible at large $q \gtrsim 1$. In the opposite limit of small $q \lesssim 0.1-1$, thermal masses affect the production rates in different and much more dramatic ways. For the $\phi q \rightarrow g q$ process, the large thermal mass of the gluon in the final state requires a highly energetic initial quark which is Boltzmann suppressed, causing the exponential drop below $q \sim 0.1$. The $\phi g \rightarrow q \bar{q}$ process however shows a strong enhancement relative to the zero-temperature limit below $q \sim 0.1$. This is more conveniently understood considering the direct process $q \bar{q} \rightarrow \phi g$. If the light $\phi$ particle emitted from the initial quark states is sufficiently soft, it becomes possible, since $m_{g}>2 m_{q}$ within the plasma, that the gluon produced from the annihilating $q \bar{q}$ pair go on-shell, which strongly enhances the $2 \rightarrow 2$ scattering amplitude. These effects of thermal masses are illustrated in Fig. 2 which shows the ratio of the reaction rates in Eq. (15), calculated with and without thermal masses. ${ }^{5}$

Second, since the gluon thermal mass is always larger than twice that of the quark, opening a new production

\footnotetext{
${ }^{5}$ In the absence of thermal masses, we regulate the forwardsingularity of $t$-channel diagrams by cutting off phase space regions where the transferred momentum is less than $\Lambda_{\mathrm{QCD}}$.
} 


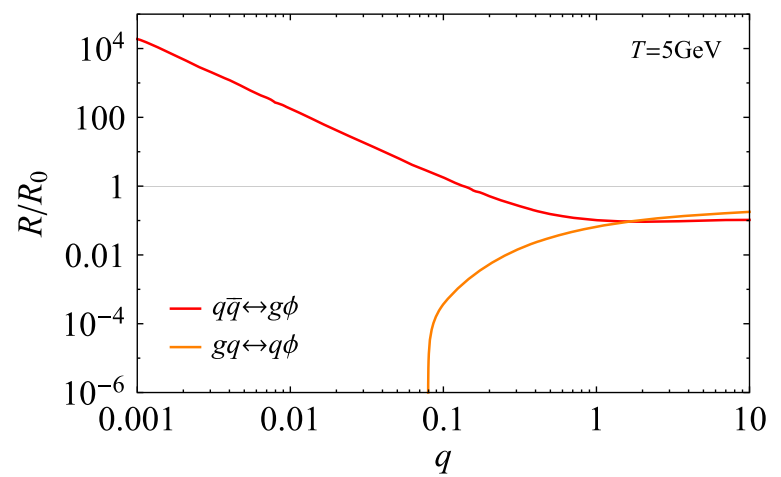

FIG. 2. Finite-temperature corrected QCD rate for $\phi$ production $(R)$ relative to their counterparts in vacuum $\left(R_{0}\right)$.

channel from the decay $g \rightarrow q \bar{q} \phi$ which is absent at zerotemperature. Note that the photon thermal mass, which emerges from QED interactions with charged particles in the plasma, is always too small to allow for the decay $\gamma \rightarrow q \bar{q} \phi$.

The impact of plasma effects on the $\phi$ distribution resulting from Eq. (6) is illustrated on Fig. 3 for $T=5 \mathrm{GeV}$, where the solid black (orange) line represents the distribution with (without) including thermal corrections. For $2 \rightarrow 2$ processes the inclusion of thermal masses suppresses by a factor of $\mathcal{O}(10)$ the production of $\phi$ particles with momentum larger than temperature. At small momenta, however, thermal masses strongly enhance $\phi$ production due to significant emission of soft $\phi$ particles together with gluons. This enhancement in the production rate allows $\phi$ to reach equilibrium at small momenta much faster than in the absence of thermal corrections since, in this case, the production peaks at momenta of $\sim T$. Consequently, the small momentum region is less populated, as shown with the orange line in Fig. 3. The gluon decay contribution is typically much smaller than the scattering contributions and quickly becomes inefficient in producing energetic $\phi$ particles due to phase space limitation. The latter contribution drops from about $30 \%$ at $p \sim T$ to roughly $10 \%$ at high momenta.

\section{Simplifying assumptions}

The full resolution of the Boltzmann equations is rather cumbersome. However a relatively accurate estimate for the relic density can be obtained upon making several simplifying assumptions. While the results presented in Figs. 4 and 5 are derived from a full calculation, we discuss below the numerical impact of such approximations.

First of all, we evaluate the impact of the choice of statistical distributions to describe particles in the plasma. The convenient assumption that particles follow a Maxwell-Boltzmann (MB) distribution is typically not justified for the freeze-in mechanism where most DM particles are produced from collisions of very relativistic particles. As shown in Fig. 3, making the approximation

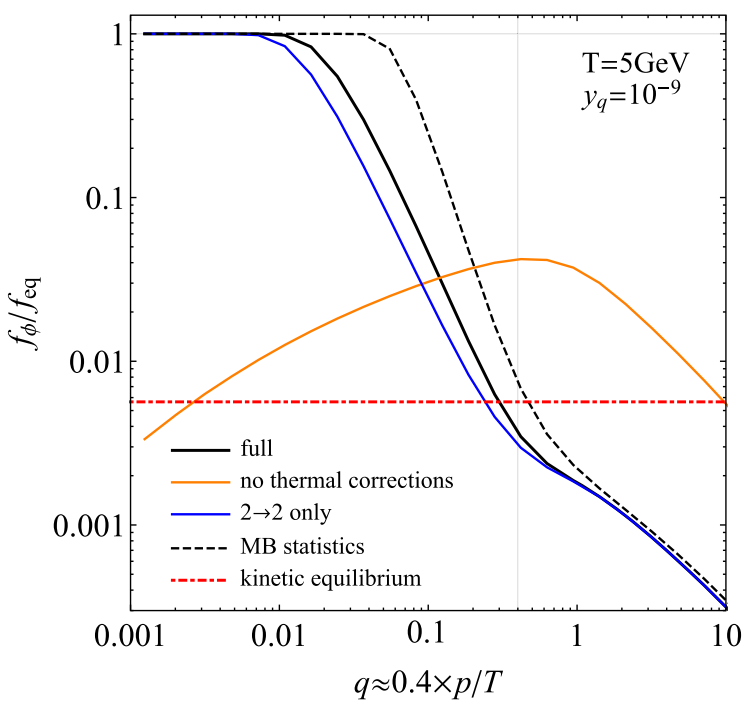

FIG. 3. Momentum distribution of the mediator normalized to the equilibrium distribution at $T=5 \mathrm{GeV}$, assuming $y_{q}=10^{-9}$. The exact treatment which includes finite temperature corrections, quantum statistical distributions and thermal gluon decays (black) is compared with the various approximations: neglecting thermal gluon decay (blue) or finite temperature corrections altogether (orange), assuming Maxwell-Boltzmann statistical distributions for all particles (dashed black) and in the kinetic equilibrium approximation (dot-dashed red). The vertical gray line denotes the typical momentum required for DM production $p \simeq m_{\chi}=T$.

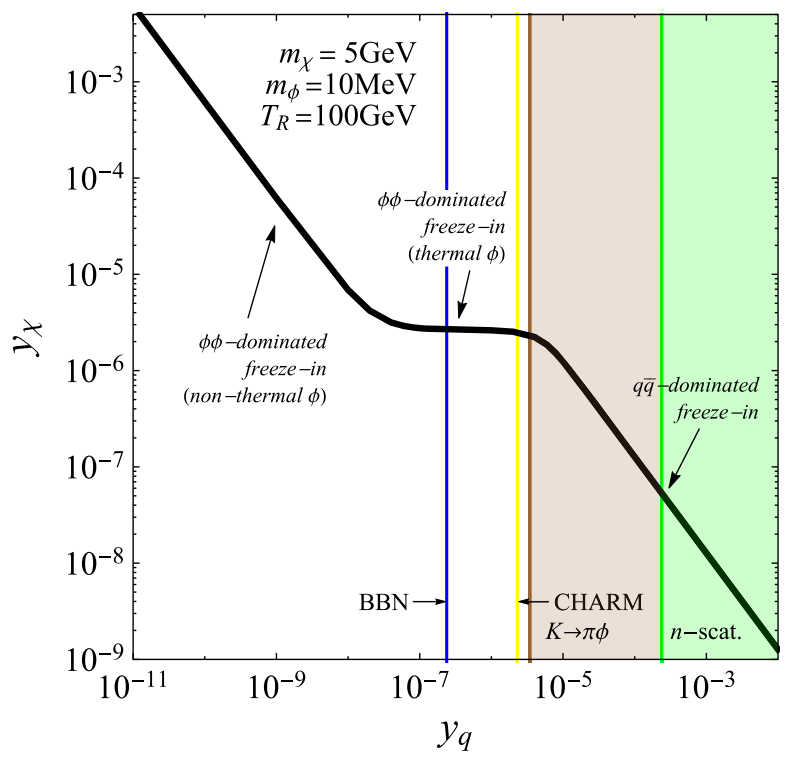

FIG. 4. Phase diagram of the relic density for a $5 \mathrm{GeV}$ mass DM and a $\mathrm{MeV}$-scale mediator. The contour in black reproduces $\Omega_{\mathrm{DM}} h^{2}=0.118$. Below (above) this line, DM is under (over) abundant. Here $y_{q}$ is defined at the scale $m_{\chi}$. The vertical bands correspond to the constraints described in Sec. VI, see text for details. 


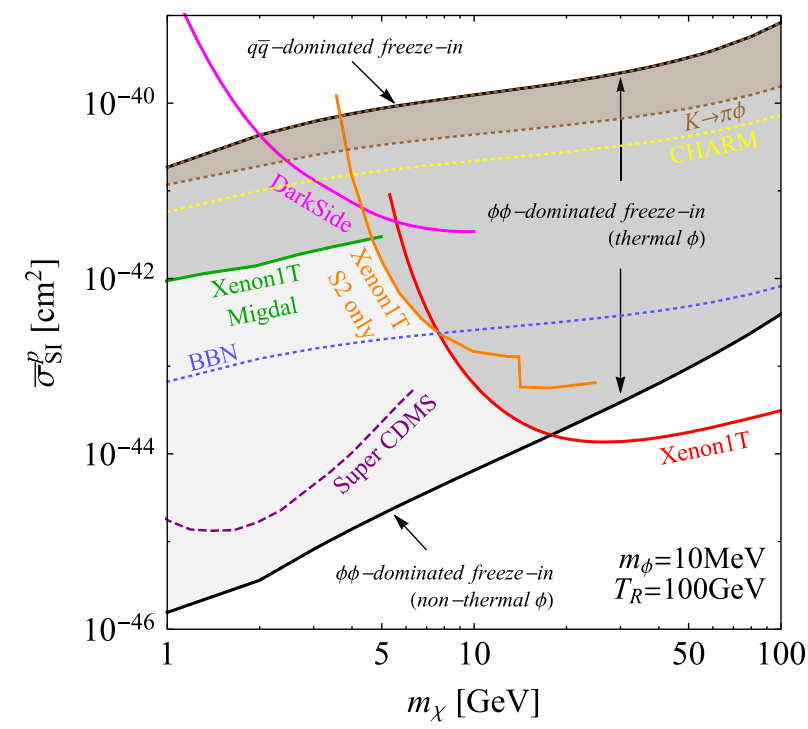

FIG. 5. Freeze-in prediction for the cross section of DM spinindependent scattering of proton at zero-momentum transfer. Experimental limits and future sensitivities have been recast to account for the $\mathrm{MeV}$-scale mediator. See text for details.

that all particles have MB distributions would overestimate the production of $\phi$ particles by more than a factor 2 for $p \approx T$ and by about $10 \%$ for much larger momenta. Note that using the MB distribution does not have a strong impact on the gluon decay contribution [42].

Second, a simple approximation would be to assume that $\phi$ is in kinetic equilibrium with the thermal bath [39]. In this case $f_{\phi} / f_{\text {eq }}$ is independent of momentum and simply given by the ratio $n_{\phi} / n_{\text {eq }}$ of the $\phi$ number density, thus avoiding having to solve the unintegrated Boltzmann equation in Eq. (8). This approximation is not justified a priori, unless $n_{\phi} \approx n_{\text {eq }}$, because there is no elastic scattering rate between $\phi$ and SM particles that is faster than the Hubble rate. Moreover, since $\phi$ production rates are faster at low momentum, the kinetic equilibrium approximation largely overestimates (underestimates) $f_{\phi}$ at high (low) momenta by several orders of magnitude, as shown in Fig. 3 with the horizontal red dot-dashed line. As argued in Appendix B the peak of DM production through fusion of out-of-equilibrium $\phi$ pairs occurs for one $\phi$ particle with a large momentum of $\mathcal{O}\left(m_{\chi}\right)$ colliding with another one nearly at rest. Therefore, within the kinetic equilibrium assumption, there is a large compensation between the $\phi$ distribution at small and large $q$. As a result of this partial cancellation the kinetic approximation allows to estimate the DM relic density from out-of-equilibrium mediator fusion within an $\mathcal{O}(1)$ factor (see below).

\section{DM PRODUCTION}

We assume negligible initial abundance for the dark sector at the end of inflation, $n_{\chi}=n_{\phi}=0$ at $T=T_{R}$ with
$T_{R}$ denoting the reheating temperature. In contrast with thermal production, the DM relic is produced by the so-called freeze-in mechanism [11,12] through feeble interactions with the thermal bath (during the radiationdominated era). There are two possible channels for DM production: $q \bar{q} \rightarrow \chi \bar{\chi}$ (with $\phi$ in the $s$-channel) and $\phi \phi \rightarrow$ $\chi \bar{\chi}$ (with $\phi$ in the $t, u$-channels) where the $\phi$ density is produced from thermal collisions of SM fields (see below).

The DM yield $Y_{\chi} \equiv n_{\chi} / s$, where $n_{\chi}$ is the DM number density and $s$ is the entropy density associated with the SM degrees of freedom, is governed by the following Boltzmann equation:

$$
s \bar{H} x \frac{d Y_{\chi}}{d x}=\gamma_{q \bar{q} \leftrightarrow \chi \bar{\chi}}+\gamma_{\phi \phi \leftrightarrow \chi \bar{\chi}},
$$

where $x \equiv m_{\chi} / T, \bar{H} \equiv H /\left[1-\left(d \log h_{\mathrm{eff}} / d \log x\right) / 3\right], H$ being the Hubble parameter, and $\gamma_{A \leftrightarrow B} \equiv \gamma_{A \rightarrow B}-\gamma_{B \rightarrow A}$. The $\gamma$ 's are the so-called (integrated) collision terms associated with the production processes described above $(A=q \bar{q}$ or $\phi \phi)$ and their depletion counterparts.

The total DM energy density today is obtained from integrating Eq. (20) between $T_{R}$ and $T_{0} \sim 2.7 \mathrm{~K}$ (the photon temperature today)

$$
\Omega_{\chi} \simeq \frac{m_{\chi} s_{0}}{\rho_{c}} \int_{x_{R}}^{x_{0}} d x \frac{d Y_{\chi}}{d x}
$$

where $s_{0} \approx 2.89 \times 10^{9} / \mathrm{m}^{-3}$ and $\rho_{c} \approx 10.54 h^{2} \mathrm{GeV} / \mathrm{m}^{-3}$ are today's entropy and critical energy densities of the Universe, respectively. $h \approx 0.674(5)$ [62] is related to the value of the Hubble parameter today as $H_{0}=100 h \mathrm{~km} / \mathrm{sec} / \mathrm{Mpc}$.

The value of $T_{R}$ is somewhat arbitrary. The simplified model under consideration being only valid below the EW scale, we set $T_{R}=100 \mathrm{GeV}$ for consistency. Higher values of $T_{R}$ would require to embed the interaction Lagrangian in Eq. (1) into a specific UV complete theory respecting the $\mathrm{SU}(2)_{L} \times U(1)_{Y}$ invariance of the SM. Note that for $m_{\chi} \ll T_{R}$, DM is dominantly produced at much lower temperatures $T \sim m_{\chi}$ where the relevant dynamics are well described by Eq. (1) and the precise value of $T_{R}$ is irrelevant. However, production of heavier DM particles would be strongly suppressed. Nevertheless, the freeze-in mechanism for $m_{\chi} \gtrsim 50 \mathrm{GeV}$ is well covered by direct detection [14] and most probably excluded by Xenon1T [63], see also Sec. V.

We solve Eq. (20) neglecting reverse processes where DM annihilates back into $q \bar{q}$ and $\phi \phi$. This is certainly a justified approximation for the hadronic channel since $\gamma_{\chi \bar{\chi} \rightarrow q \bar{q}} / \gamma_{q \bar{q} \rightarrow \chi \bar{\chi}} \sim \mathcal{O}\left(n_{\chi}^{2} / n_{\mathrm{eq}}^{2}\right)$ and $n_{\chi} \ll n_{\mathrm{eq}}$ at all times. The situation is less clear for mediator channel though, in particular, because, as we show below, DM could be efficiently produced also in the case that $\phi$ is not in equilibrium with the thermal bath and $n_{\phi} \ll n_{\text {eq }}$. We verified numerically that the number density $\bar{n}_{\phi}$ of $\phi$ particles with 
energy above $m_{\chi}$ is larger than $n_{\chi}$ by a factor of $\mathcal{O}\left(10^{3}\right)$ or more in regions of parameter space where $\phi \phi \rightarrow \chi \bar{\chi}$ is dominant. Hence $\gamma_{\chi \bar{\chi} \rightarrow \phi \phi} / \gamma_{\phi \phi \rightarrow \chi \bar{\chi}} \sim \mathcal{O}\left(n_{\chi}^{2} / \bar{n}_{\phi}^{2}\right) \ll 1$ whenever relevant and the reverse process is also negligible in this case.

For illustration, we show in Fig. 4 the "phase diagram" in the $y_{q}-y_{\chi}$ coupling plane resulting from the calculation of the relic density of $5 \mathrm{GeV}$ mass DM in the model described in the previous section. One distinguishes three different regimes for DM production depending on the value of the quark-mediator coupling.

For relatively large values of $y_{q}$ the dominant DM production mechanism is directly from collisions of thermal SM particles, through $q \bar{q} \rightarrow \chi \bar{\chi}$ whose cross section (as well as $\Omega_{\chi}$ ) scales like $\left(y_{q} y_{\chi}\right)^{2}$.

As the quark coupling is decreased, SM collisions are less and less frequently producing DM particles and below a critical value of $y_{q}^{\text {crit }}$ (the precise value slightly varies with $m_{\chi}$ ) collisions of mediator particles become the dominant production mechanism. Since $\phi$ is assumed with negligible initial density, the efficiency of this process is determined by how much $\phi$ particles are produced from SM collisions. For values not too far below $y_{q}^{\text {crit }}$, the quark coupling is typically still sufficiently large so that the mediator reaches equilibrium with the thermal bath before DM production effectively starts at $T \sim m_{\chi}$. In that case, the density of $\phi$ no longer depends on $y_{q}$ and $\Omega_{\chi}$ scales like $y_{\chi}^{4}$. Hence the plateau in the phase diagram of Fig. 4.

For even smaller values of $y_{q} \lesssim y_{q}^{\mathrm{eq}}$, where $y_{q}^{\mathrm{eq}}$ is the minimal coupling value needed to keep $\phi$ in thermal equilibrium with $\mathrm{SM}$, the $\phi$ production rate is too slow, such that the mediator is out-of-equilibrium during DM production. In this case, the $\phi \phi \rightarrow \chi \bar{\chi}$ rate is suppressed by the (square of the) small density of nonthermal $\phi$. Nevertheless, this mechanism still dominates over direct production from SM collisions. This is understood as follows. Far from equilibrium, i.e., for $y_{q} \ll y_{q}^{\mathrm{eq}}$, the momentum distribution of $\phi$ is proportional to the production rate and scales as $f_{\phi} \sim\left(y_{q} / y_{q}^{\mathrm{eq}}\right)^{2} f_{\text {eq }}$ since the rate is dominated by single production processes. As a result, the $\phi \phi \rightarrow \chi \bar{\chi}$ contribution to the relic density scales as $\left(y_{q} y_{\chi} / y_{q}^{\mathrm{eq}}\right)^{4}$ in this regime, and the ratio of collision terms in Eq. (20) is (roughly) $\gamma_{\phi \phi \rightarrow x \bar{\chi}} / \gamma_{q \bar{q} \rightarrow \chi \bar{\chi}} \sim\left(y_{q} y_{\chi}\right)^{2} /\left(y_{q}^{\mathrm{eq}}\right)^{4}$. The $q \bar{q}$-dominated freeze-in mechanism requires $y_{q} y_{\chi} \sim$ $10^{-11}$ in order to reproduce the observed DM relic density, while typically $y_{q}^{\mathrm{eq}} \approx 10^{-7}$. Hence, the freeze-in production of DM from collisions of nonthermal $\phi$ particles dominates over the direct contribution from SM collisions by a factor of $\sim 10^{5}$.

To conclude this section, we stress the importance of using the full solution of the Boltzmann equation for $\phi$ in computing the DM relic density. Detailed comparison reveals that assuming a kinetic equilibrium distribution for the mediators overestimates $\Omega_{\chi}$ by a factor $\sim 2$ for $y_{q}<$ $10^{-9}$ while the discrepancy with the full calculation rapidly disappears for coupling values large enough so that $\phi$ approaches thermal equilibrium. As noted in Sec. III, using Maxwell-Boltzmann distributions leads to an overproduction of $\phi$ particles. For $m_{\chi}=5 \mathrm{GeV}$, this overestimates the relic density by about $50 \%$ in a regime where $\phi$ is out-ofequilibrium. The neglect of finite temperature corrections and plasma effects also leads to an overproduction of $\phi$ particles that yields an $\mathcal{O}(1)$ increase in the relic density. On the other hand, if $\phi$ particles are in thermal equilibrium, the relic density is roughly $40 \%$ higher when using the Bose-Einstein statistical distribution. Finally, when DM is mainly produced from $u$-quarks, its relic density increases more mildly, around 25\%, when using a MB distribution.

\section{PREDICTIONS FOR DIRECT DETECTION EXPERIMENTS}

A relic of DM particles of mass above the $\mathrm{GeV}$-scale can be directly detected by observing scattering events on heavy nuclei [1]. For a scalar mediator the expected signal is spin-independent (SI) with differential rate $d R / d E_{R}$ as a function of the nuclear recoil energy $E_{R}$ given by [64],

$$
\frac{d R}{d E_{R}}=\frac{\rho_{0} \bar{\sigma}_{\mathrm{SI}} N_{A}}{\sqrt{\pi} v_{0} m_{\chi} \mu_{\chi N}^{2}} F^{2}(q) \eta\left(q^{2}\right) \times \frac{m_{\phi}^{4}}{\left(q^{2}+m_{\phi}^{2}\right)^{2}},
$$

where $q^{2} \equiv \sqrt{2 m_{N} E_{R}}$ is the momentum transferred, $\rho_{0}=$ $0.3 \mathrm{GeV} / \mathrm{cm}^{3}$ is the DM energy density today, $m_{N}$ is the mass of the target nucleus, $\mu_{\chi N} \equiv m_{\chi} m_{N} /\left(m_{\chi}+m_{N}\right)$ is the reduced mass of the DM-nucleus system and $N_{A}$ is the Avogadro constant. $F(q)$ is a nuclear form factor that describes the loss of coherence among nucleons at finite momentum transfer, while $\eta\left(q^{2}\right)$ captures the dependence on the DM velocity distribution. Their explicit functions are given in Appendix C.

$\bar{\sigma}_{\mathrm{SI}}$ is the SI DM-nucleus scattering cross section evaluated at zero momentum transfer. In the limit of isospin symmetry it is related to the cross section on a single nucleon, say proton, as $\bar{\sigma}_{\mathrm{SI}} / \mu_{\chi N}^{2}=A^{2} \bar{\sigma}_{\mathrm{SI}}^{p} / \mu_{\chi p}^{2}$ where $A$ is the total number of nucleons in the target and $\mu_{\chi p}=$ $m_{\chi} m_{p} /\left(m_{\chi}+m_{p}\right)$ is the reduced mass of DM and the proton. In our simplified model, assuming $m_{\phi} \ll m_{\chi}$, we have

$$
\bar{\sigma}_{\mathrm{SI}}^{p}=\frac{y_{p}^{2} y_{\chi}^{2} \mu_{\chi p}^{2}}{\pi m_{\phi}^{4}},
$$

where $y_{p}$ is defined in Eq. (3). Finally, the last term on the right-hand side of Eq. (22) parametrizes the $t$-channel propagator of the mediator. For $q^{2} \ll m_{\phi}^{2}$, the DM-nucleus scattering is well described by a contact interaction, which is the implicit assumption behind the limit on $\bar{\sigma}_{\mathrm{SI}}^{p}$ (as a 
function of $m_{\chi}$ ) presented by all DD experiments. However for $m_{\phi} \lesssim q_{\max } \sim \mathcal{O}(\mathrm{GeV})$, the $q^{2}$-dependence of the cross section is not negligible, and limits assuming contact interactions no longer apply. Nonetheless, the DD sensitivity for light mediators can be estimated by recasting existing limits based on the event rate expected from DM scattering in a given experiment. We have followed the recasting procedure of micrOMEGAs [65] for Xenon1T [63] and DarkSide50 [66]. In the low mass region, the latter is superseded by two analyses from Xenon1T using the S2 signal only [67] and taking advantage of the Migdal effect [68]. To estimate the projected sensitivity of SuperCDMS [69], the expected event rate is computed using

$$
R=\int d E_{R} \epsilon\left(E_{R}\right) \frac{d R}{d E_{R}},
$$

where $\epsilon\left(E_{R}\right)$ denotes the detection efficiency. We assume that the efficiency vanishes below the nuclear energy threshold of $0.04 \mathrm{keV}$ and increases linearly to reach $85 \%$ at $2 \mathrm{keV}$, for higher energies we take a constant efficiency [69]. Note that the exact shape of the efficiency curve at low nuclear recoil energies strongly affects the event rate, since the energy distribution for a light mediator peaks at low energies.

The freeze-in prediction for the SI cross section strongly depends on whether the relic abundance is dominated by $q \bar{q}$-initiated or $\phi \phi$-initiated collisions in the early Universe. In the first case, both $\Omega_{\chi}$ and $\bar{\sigma}_{\mathrm{SI}}^{p}$ depend on the same combination of couplings, $\left(y_{q} y_{\chi}\right)^{2}$, such that the relic density uniquely determines the direct detection signal for a fixed DM mass. This prediction is represented by the upper black line on Fig. 5. In the second case, when mediator collisions dominate the freeze-in production of DM, the product of couplings $y_{q} y_{\chi}$ could be much smaller, and its value depends on whether the mediator is in thermal equilibrium with the SM or not, see Fig. 4. When freeze-in is dominated by nonthermal $\phi, \Omega_{\chi} \propto\left(y_{q} y_{\chi}\right)^{4}$ and the product $y_{q} y_{\chi}$ is also fixed. Thus, the relic density also makes a unique prediction for $\bar{\sigma}_{\mathrm{SI}}^{p}$ in this case, which is represented by the lower black line on Fig. 5. Conversely, when the mediator is in thermal equilibrium during DM production, $\Omega \propto y_{\chi}^{4}$ while the quark coupling is in the range $y_{q}^{\mathrm{eq}} \lesssim y_{q} \lesssim y_{q}^{\text {crit }}$. Hence, the direct detection cross section predicted by the relic density is not unique, but rather lies within the entire interval between the predicted value of $q \bar{q}$ dominated regime (above) and that of the $\phi \phi$-dominated one with nonthermal $\phi$ (below). This results in the grayshaded band shown in Fig. 5 which approximately spans three to five orders of magnitude, depending on the DM mass. Note that the $\bar{\sigma}_{\mathrm{SI}}^{p}$ range predicted by freeze-in is narrower for larger values of $m_{\chi}$, which is simply due to the fact that the mediator requires a larger coupling to the SM in order to reach equilibrium before DM production starts.
The predicted direct detection signals are already well covered by existing experiments. In particular, the current limits from Xenon1T rule out the parameter space consistent with freeze-in for DM masses above $30 \mathrm{GeV}$ and partly covers the $\phi \phi$-dominated freeze-in down to its threshold sensitivity, corresponding to $m_{\chi} \approx 6 \mathrm{GeV}$. For lower DM masses, the new analyses by Xenon1T based on the Migdal effect or exploiting the S2 signal only exclude the $q \bar{q}$-dominated freeze-in as well as part of the parameter space of the $\phi \phi$-dominated regime. This region is also excluded partly by DarkSide50. Moreover, future experiments will significantly improve the coverage for light DM. For instance, the projected reach for SuperCDMS will allow to probe a significant fraction of the freeze-in prediction below $5 \mathrm{GeV}$.

Finally, several terrestrial, astrophysics and cosmology constraints, which we summarize in the next section for completeness, can be imposed on a MeV-scale hadrophilic scalar. Imposing all these constraints at face value for $m_{\phi}=$ $10 \mathrm{MeV}$ severely restricts the range for the quark-mediator coupling, such that most of the mediator-dominated regime would be excluded. The narrower band of the SI cross sections that reproduce the relic abundance and satisfy these constraints corresponds to the upper part of the light grey area above the BBN line in Fig. 5. This region is significantly enlarged to the whole light grey area when the $\mathrm{BBN}$ bound is evaded in the presence of an additional decay channel for the mediator into neutrinos.

\section{OTHER CONSTRAINTS}

Other constraints exist on individual couplings of a light mediator to SM quarks, both from terrestrial experiments and astrophysics and cosmological observations. See Refs. [33,43] for detailed reviews. For completeness we quickly describe below the constraints shown in Figs. 4 and 5 that are relevant to our scenario.

Several laboratory experiments are sensitive to light particles coupled to quarks. Neutron-scattering experiments at low energies are sensitive to the coupling of $\phi$ to neutrons. The strongest constraint for a $10 \mathrm{MeV}$ mass [70] arises from analyzing the momentum distribution of $\mathrm{keV}$-scale neutrons scattered off lead nuclei, giving $y_{n} \lesssim$ $1.5 \times 10^{-3}$ [71]. This bound translates to $y_{q}(2 \mathrm{GeV}) \lesssim$ $2.9 \times 10^{-4}$ for a scalar coupled to $u$-quark only $[48,49]$.

Additional constraints come from rare meson decays. Light mediators coupled to quarks can be produced onshell in $B \rightarrow K \phi$ and $K \rightarrow \pi \phi$ decays [72]. For the coupling values we envisage here $\phi$ is stable on collider scales and would appear as missing energy in the decays. Due to the heavy bottom mass, the $B \rightarrow K$ transition is induced at one-loop by an electroweak penguin diagram. This penguin is suppressed by the light $u$-quark mass and small CKM matrix elements $V_{u s} V_{u b}$, which makes it negligible given the current experimental bound on such 
decay [73]. However, the $K \rightarrow \pi$ transition receives a less suppressed tree-level contribution from the chiral Lagrangian [43]. The limit on the $\operatorname{BR}\left(K^{+} \rightarrow \pi^{+} \nu \bar{\nu}\right)$ [74] provides a strong constraint from meson decays, yielding $y_{q}(2 \mathrm{GeV}) \lesssim 4.2 \times 10^{-6}$ for $u$-quark. The forthcoming NA62 experiment is expected to improve the sensitivity in the $K^{+} \rightarrow \pi^{+} \nu \bar{\nu}$ channel by a factor of $\sim 3$ [75]. Light mediators coupled to quarks are also constrained from proton beam dump experiments. In particular, the axionlike particle search at the $400 \mathrm{GeV}$ SPS by the CHARM collaboration [76] can be used to constrain the process $\eta \rightarrow$ $\pi \phi$ where $\phi$ decay into two photons [43]. This search yields the strongest upper limit from meson decay, $y_{q}(2 \mathrm{GeV}) \lesssim$ $2.8 \times 10^{-6}$ for $u$-quark. Note however that this constraint can be relaxed if $\phi$ is allowed to decay into an invisible channel, like neutrinos.

Light bosons coupled to nucleons can be emitted in stars. Below a critical coupling value, the emitted bosons interact so weakly with the stellar medium that they escape the star without being reabsorbed, thus contributing to its cooling. Lack of evidence of such additional energy loss mechanisms in several stellar systems thus constrains the coupling of light bosons [77]. For large enough couplings, the new bosons are efficiently reabsorbed and trapped in the stellar medium, thus only contributing to energy losses from the outermost shell [78]. Horizontal branch and red giant stars are too cold to emit $10 \mathrm{MeV}$-scale bosons. Those can however be constrained from supernova 1987A (SN1987A) whose temperature reached $T \sim 30 \mathrm{MeV}$. Stellar cooling constraints from SN1987a were extensively studied for pseudoscalar particles, notably axions; see e.g., Ref. [79] for a recent analysis both in the free-streaming and the trapping regimes. Transposing naively axion bounds to the case of scalar particles [80] would yield the following upper bound on the $u$-quark coupling, $y_{q}(2 \mathrm{GeV}) \lesssim 4.2 \times 10^{-11}$, and thus exclude most of the coupling range relevant to the sequential freeze-in mechanism.

Light bosons with tiny coupling to SM fields typically live long enough to leave traces in well-understood late cosmological phenomena, such as BBN or the cosmic microwave background $(\mathrm{CMB})$. If the mediator survives until $\mathrm{BBN}$, its $\phi \rightarrow \gamma \gamma$ decay could inject electromagnetic energy in the thermal bath, hence increasing its entropy density and (if the decay products are sufficiently energetic) dissociating the freshly formed light elements. Given its very small scattering cross section with the SM, the mediator will decouple relativistically from the thermal bath at $T \sim m_{\phi}=10 \mathrm{MeV}$. Then, in order to avoid strong alteration of the standard BBN predictions for the abundances of light elements, its relic must decay away before the onset of the first nuclear reactions at $t \simeq 1 \mathrm{sec}$. From Eq. (5), this implies $y_{q}(2 \mathrm{GeV}) \gtrsim 2.9 \times 10^{-7}$. Note that this constraint can be evaded by shortening the lifetime of the mediator through either increasing its mass or opening an additional decay channel into neutrinos. In the latter case, we checked, using the alterBBN [81] code, that the neutrinos produced from the decay of a $10 \mathrm{MeV}$ mediator thermalize before neutrino decoupling and do not spoil $\mathrm{BBN}$ predictions.

\section{DISCUSSION}

In the above analysis, we concentrated on the specific case of a $10 \mathrm{MeV}$ scalar mediator that couples to DM and $u$-quarks. We also implicitly assumed a coupling to SM neutrinos whenever necessary to avoid cosmological constraints. However, the mechanism of DM freeze-in production from fusion of out-of-equilibrium mediators is more generic. First of all, our results for the DM relic density would equally apply in cases where $\phi$ couples to any of the light quark flavors. Qualitatively similar results would apply also for leptophilic mediators, although in that case large portal couplings are typically required since the mediator density is now dominantly created by electroweak interactions, rather than QCD. Moreover, DM phenomenology remains valid as long as the mediator mass is below $\sim 100 \mathrm{MeV}$. Indeed such a light mediator has little impact on the relic density, since $m_{\chi} \gg m_{\phi}$, as well as on predictions and limits from direct detection, since the scaling of the cross section $\bar{\sigma}_{S I}^{p} \propto m_{\phi}^{-4}$ holds in this mass range. The mass of the mediator and its possibility to decay into a neutrino channel do, however, affect other constraints, most notably those from BBN. In the absence of the neutrino channel, a mediator's lifetime $\tau_{\phi} \lesssim 1$ sec can be achieved with a large enough coupling $y_{q}$, more precisely for $y_{q}\left(m_{\phi} / 10 \mathrm{MeV}\right)^{3 / 2}>2.9 \times 10^{-7}$. While BBN constraints restrict the out-of-equilibrium regime for lighter mediators, $m_{\phi}>30 \mathrm{MeV}$ allows for sufficiently smaller values of $y_{q}$ such that the out-ofequilibrium regime opens up significantly. This also implies a wider region in direct detection of light dark matter, with $m_{\chi} \lesssim 6 \mathrm{GeV}$, that is free of constraints. Note also that for $m_{\phi}>30 \mathrm{MeV}$, the supernova constraint disappears thus further relaxing constraints on the whole out-of-equilibrium window.

In summary, we performed a detailed calculation of DM production via the production of out-of-equilibrium mediators by solving the unintegrated Boltzmann equation for the latter, instead of making the kinetic equilibrium approximation, and including the effect of thermal masses and of quantum statistics. Each of these effects has a large impact on the prediction of the dark matter relic density. We also showed using a simplified model that while this mechanism faces cosmological constraint it can be probed by direct detection experiments. Increasing the sensitivity of direct detection experiments at low masses is however crucial to completely cover the pure freeze-in via out-of-equilibrium regions in the future. 


\section{ACKNOWLEDGMENTS}

We thank T. Hambye and M. Tytgat for fruitful discussions and A. Arbey for providing useful information on alterBBN. This work was funded by RFBR and CNRS, project No. 20-52-15005 and by a grant AAP-USMB. G. B. and A. P. would like to thank the Paris-Saclay Particle Symposium 2019 with the support of the P2I and SPU research departments and of the P2IO Laboratory of Excellence (program "Investissements d'avenir" ANR-11IDEX-0003-01 Paris-Saclay and ANR-10-LABX-0038) for their hospitality and support during the completion of this work. B.Z. is supported by the Programa Atracción de Talento de la Comunidad de Madrid under Grant No. 2017-T2/TIC-5455, from the Comunidad de Madrid/ UAM "Proyecto de Jóvenes Investigadores" Grant no. SI1/ PJI/2019-00294, from Spanish "Proyectos de I+D de Generacion de Conocimiento" via grants PGC2018096646-A-I00 and PGC2018-095161-B-I00.

\section{APPENDIX A: REACTION RATE INTEGRALS FOR MEDIATOR PRODUCTION}

We elaborate on the calculation of the reaction rates relevant for mediator production in the early Universe.

\section{Scattering}

The rate of the $2 \rightarrow 2$ scattering reaction $12 \leftrightarrow 3 \phi$ is

$$
R_{\text {scat }}=\frac{g_{3}}{(2 \pi)^{3}} \int d^{3} p_{3} f_{3} \sigma_{3 \phi \rightarrow 12} v_{\mathrm{M} \varnothing \mathrm{l}},
$$

see Eq. (15). The Møller velocity $v_{\mathrm{M} ø l}=F /\left(E E_{3}\right)$ where $F=\sqrt{s} p_{*}(s)$ is the (Lorentz-invariant) flux of incoming particles, expressed in terms of the total energy $\sqrt{s}$ and the $\phi$ momentum $p_{*}(s)=\sqrt{s} / 2\left[1-\left(m_{3}+m_{\phi}\right)^{2} / s\right]^{1 / 2}[1-$ $\left.\left(m_{3}-m_{\phi}\right)^{2} / s\right]^{1 / 2}$ in the center-of-mass frame, while $E$ and $E_{3}$ are the energies of $\phi$ and particle 3 in the plasma frame. The differential element in Eq. (A1) writes $d^{3} p_{3}=$ $2 \pi p_{3}^{2} d p_{3} d \cos \theta$ where $\cos \theta$ can be identified to the angle between $p_{3}$ and $p$ in the plasma frame. Using $s=m_{3}^{2}+m_{\phi}^{2}+2\left(E E_{3}-p p_{3} \cos \theta\right)$, the $\cos \theta$ integral can be traded for an integral over $s$, yielding

$$
R_{\text {scat }}=\frac{g_{3}}{8 \pi^{2} E p} \int d s \sqrt{s} p_{*} \sigma_{3 \phi \rightarrow 12} \int d E_{3} f_{3},
$$

where $s \geq \max \left[\left(m_{1}+m_{2}\right)^{2},\left(m_{3}+m_{\phi}\right)^{2}\right]$. The boundary values $E_{3}^{\min , \max }=E_{3}^{\min , \max }(s, E)$ are obtained as follows. For fixed $s$ and $E, E_{3}$ reaches its extremal values when particle 3 and $\phi$ are collinear. In this case, $E_{3}$ relates to $s$ and $E$ through a Lorentz boost transformation,

$$
E_{3}=E_{3 *} \cosh (y)-p_{*} \sinh (y),
$$

where $E_{3 *}=\left(s+m_{3}^{2}-m_{\phi}^{2}\right) / 2 \sqrt{s}$ and $p_{3 *}=-p_{*}$ are the energy and momentum of particle 3 in the center-of-mass frame and $y$ is the rapidity of the center-of-mass, which satisfies $E=E_{*} \cosh (y)+p_{*} \sinh (y)$ with $E_{*}=\sqrt{s}-E_{3 *}$. There are two independent solutions,

$$
y_{ \pm}=\log \left(\frac{E \pm p}{E_{*}+p_{*}}\right)
$$

where $y_{+}\left(y_{-}\right)$is reached when the momenta of particle 3 and $\phi$ are parallel (antiparallel), and the energy of particle 3 in the plasma frame is $E_{3}^{\max }\left(E_{3}^{\min }\right)$.

\section{Decay}

The rate of the 3-body decay reaction $1 \rightarrow 23 \phi$ is

$$
R_{\text {decay }}=g_{1} e^{E / T} \int d^{3} p_{1} f_{1} \frac{d \Gamma_{1 \rightarrow 23 \phi}}{d^{3} p},
$$

see Eq. (16), where $\Gamma_{1 \rightarrow 23 \phi}$ is the decay width of particle 1 in the plasma frame, $p$ is the $\phi$ momentum in that frame and

$$
\frac{d \Gamma_{1 \rightarrow 23 \phi}}{d^{3} p}=\frac{1}{2 \pi E p} \frac{d \Gamma_{1 \rightarrow 23 \phi}}{d E} .
$$

In order to determine $d \Gamma_{1 \rightarrow 23 \phi} / d E$, we will first calculate the differential width in the rest frame of the decaying particle $1, d \Gamma_{1 \rightarrow 23 \phi}^{*} / d E_{*}$, and boost it to plasma frame. First of all, the width is boosted by a Lorentz factor $d \Gamma_{1 \rightarrow 23 \phi}=m_{1} / E_{1} \times d \Gamma_{1 \rightarrow 23 \phi}^{*}$. Then, one needs to find how many $\phi$ particles with energy $E_{*}$ in the rest frame of particle 1 wind up with energy $E$ in the plasma frame. The energies $E$ and $E_{*}$ are related by a boost transformation

$$
E=E_{*} \cosh (y)-p_{*} \cos \theta_{*} \sinh (y),
$$

with rapidity $y=1 / 2 \log \left[\left(E_{1}+p_{1}\right) /\left(E_{1}-p_{1}\right)\right] . \theta_{*}$ is the angle between the $\phi$ momentum in the particle 1 rest frame and the boost direction, given by particle 1 momentum. Assuming the differential width is flat in $\cos \theta_{*}$, which is the case for the decay of a scalar or an unpolarized particle with nonzero spin, a $\delta$-distribution $d \Gamma / d E_{*}=A \delta\left(E_{*}-\right.$ $\left.E_{0 *}\right)$ in the particle 1 rest frame yields a rectangular function in terms of $E$ in the plasma frame, $d \Gamma / d E=$ $A / N\left[\Theta\left(E-E_{\min }\right)-\Theta\left(E-E_{\max }\right)\right]$ whose boundaries are defined by Eq. (A7) upon setting $\cos \theta_{*}= \pm 1$ and normalization is rescaled by the size of the rectangle $N=E_{\max }-E_{\min }=2\left(E_{0 *}^{2}-m_{\phi}^{2}\right)^{1 / 2} \sinh (y)$. Since any generic spectrum in $E_{*}$ can be decomposed as a (infinite) set of $\delta$-functions peaking at different values of $E_{*}$, the boosted spectrum is simply given by adding up the rectangles, yielding 


$$
\frac{d \Gamma_{1 \rightarrow 23 \phi}}{d E}=\frac{m_{1} / E_{1}}{2 \sinh (y)} \int \frac{d E_{*}}{\sqrt{E_{*}^{2}-m_{\phi}^{2}}} \frac{d \Gamma_{1 \rightarrow 23 \phi}^{*}}{d E_{*}},
$$

where the integration boundaries are $E \cosh (y)-p \sinh (y) \leq$ $E_{*} \leq E \cosh (y)+p \sinh (y)$.

\section{APPENDIX B: APPROXIMATE $f_{\phi}$ SOLUTIONS}

We derive analytical solutions for $f_{\phi}$ within some simple approximations. DM is dominantly produced at temperature $T \sim m_{\chi} / 3$, from collisions of very relativistic $\phi$ particles with momentum $p \gtrsim m_{\chi} \gg m_{\phi}$, which corresponds to $q \gtrsim 1$.

Consider the reaction rate for $2 \rightarrow 2$ processes of Eq. (A2) for large momentum $p \gg m_{\phi}, m_{3} \sim g_{s} T$. In that limit, $\exp \left(y_{-}\right) \simeq 0$ and $\exp \left(y_{+}\right) \simeq 2 p / \sqrt{s}$ such that $E_{3} \gtrsim s /(4 p)$. Assuming Maxwell-Boltzmann statistics for SM particles in the plasma, $f_{3}=\exp \left(-E_{3} / T\right)$ gives $\int d E_{3} f_{3} \simeq T \exp [-s /(4 p T)]$. For high-energy scattering in the $t$-channel $\sigma_{3 \phi \rightarrow 12} \sim c / s \log \left(s / m_{0}^{2}\right)$, where $c \sim g_{s}^{2} y_{q}^{2} \ll 1$ and $m_{0} \sim g_{s} T$ is the mass of the exchanged particle, and

$$
R_{2 \rightarrow 2} \sim \frac{c T^{2}}{p} \log \left(\frac{p T}{m_{0}^{2}}\right)
$$

Hence the reaction rate is typically slower the larger $p$, with an approximate scaling $R_{2 \rightarrow 2} \propto 1 / p$. Since $H \sim T^{2} / M_{\mathrm{Pl}}$ in the radiation-dominated era, the solution to the Boltzmann equation in Eq. (8) is approximately of the form

$$
f_{\phi}(p, T) / f_{\mathrm{eq}} \sim 1-\exp \left[-\frac{c M_{\mathrm{Pl}}}{p}\left(1+\log \frac{p}{T}\right)\right] .
$$

At a given temperature, the density of $\phi$ particles drops faster below $f_{\text {eq }}$ the larger the momentum. As a result, it takes more time for fast particles to reach equilibrium.

The approximate solution in Eq. (B2) proves useful for identifying which region(s) of the momentum distribution is relevant for DM production. The integrand of the collision term of the $\phi \phi \rightarrow \bar{\chi} \chi$ process involves the product $\eta \equiv f_{\phi}\left(p_{1}\right) f_{\phi}\left(p_{2}\right)$ where $p_{1,2}$ are the momenta of the two colliding $\phi$ particles. If $\phi$ were in equilibrium with the SM, assuming Maxwell-Boltzmann statistics, the above product would only depend on the total momentum, $\eta_{\text {eq }}=f_{\text {eq }}\left(p_{1}\right) f_{\text {eq }}\left(p_{2}\right)=\exp \left(-p_{+}\right)$with $p_{+} \equiv p_{1}+p_{2}$, and all possible repartitions of $p_{+}$between $p_{1}$ and $p_{2}$ would equally contribute. This is not necessarily the case for $\phi$ particles out-of-equilibrium. Consider the distribution of Eq. (B2) in evaluating $\eta$ in two distinct regimes where both particles have comparable momenta $p_{1} \sim p_{2} \sim p_{+} / 2$, or one particle carries all the total required momentum, $p_{1} \sim p_{+}$and $p_{2} \sim 0$. In the first regime, $\eta / \eta_{\mathrm{eq}} \sim[1-$ $\left.\exp \left(-2 c M_{\mathrm{Pl}} / p_{+}\right)\right]^{2} \sim\left(2 c M_{\mathrm{Pl}} / p_{+}\right)^{2}$, where in the last expression we used $p_{+} \gg c M_{\mathrm{Pl}}$, typically valid outof-equilibrium. Conversely, in the second regime the suppression is less severe, $\eta / \eta_{\mathrm{eq}} \sim 1-\exp \left(-c M_{\mathrm{Pl}} / p_{+}\right) \sim$ $c M_{\mathrm{Pl}} / p_{+}$, because slow particles are close to equilibrium. Hence, production of heavy DM from much lighter, out-ofequilibrium $\phi$ fusion is dominated by fast particles colliding slow ones.

\section{APPENDIX C: RELEVANT FORMULAS FOR DIRECT DETECTION}

We parametrize the nuclear form-factor with a spherically symmetric Fermi distribution, $F(q)=c \int r^{2} d r e^{-i q r} /$ $\left[1+\exp \left[\left(r-R_{A}\right) / a\right]\right]$ where the parameter $R_{A}=\left(1.23 A^{1 / 3}-\right.$ $0.6) \mathrm{fm}$ ( $A$ denoting the mass number of the nucleus) and the surface thickness $a=0.52 \mathrm{fm}$ have been extracted from muon scattering data for various nuclei $[64,82]$. The normalization constant $c$ is such that $F(q=0)=1$.

We assume the velocity distribution of DM is a Maxwellian centered on $v_{0}=220 \mathrm{~km} / \mathrm{sec}$, the galactic velocity. Further including the effects of both the velocity of the Earth relative to the galactic rest frame $v_{E}=$ $232 \mathrm{~km} / \mathrm{sec}$ and the galactic escape velocity $v_{\mathrm{esc}}=$ $544 \mathrm{~km} / \mathrm{sec}$ yields for the velocity distribution in Eq. (22) [64]

$\eta\left(q^{2}\right)=\frac{k_{0}}{k_{1}}\left[\frac{\sqrt{\pi} v_{0}}{4 v_{E}}\left(\operatorname{erf}_{+}-\operatorname{erf}_{-}\right)-\delta \exp \left(-\frac{v_{\text {esc }}^{2}}{v_{0}^{2}}\right)\right]$,

where $k_{1} / k_{0} \equiv \operatorname{erf}\left(v_{\text {esc }} / v_{0}\right)-2 v_{\text {esc }} /\left(\sqrt{\pi} v_{0}\right) \exp \left(-v_{\text {esc }}^{2} / v_{0}^{2}\right)$, $\operatorname{erf}_{ \pm} \equiv \operatorname{erf}\left(v_{ \pm} / v_{0}\right), \delta \equiv\left(v_{+}-v_{-}\right) / 2 v_{E}$ with $v_{ \pm}=v_{\min } \pm$ $v_{E}$ if $v_{\min }<v_{\text {esc }} \mp v_{E}$ and $v_{ \pm}=v_{\text {esc }}$; otherwise, $v_{\min }=$ $q /\left(2 \mu_{\chi T}\right)$ and erf denotes the error function.
[1] M. W. Goodman and E. Witten, Detectability of certain dark matter candidates, Phys. Rev. D 31, 3059 (1985); 31, 325 (1984).

[2] J. Alexander et al., Dark Sectors 2016 Workshop: Community Report (2016), arXiv:1608.08632, http://lss.fnal .gov/archive/2016/conf/fermilab-conf-16-421.pdf.
[3] Y. Hochberg, T. Lin, and K. M. Zurek, Detecting ultralight bosonic dark matter via absorption in superconductors, Phys. Rev. D 94, 015019 (2016).

[4] Y. Hochberg, T. Lin, and K. M. Zurek, Absorption of light dark matter in semiconductors, Phys. Rev. D 95, 023013 (2017). 
[5] M. Battaglieri et al., in U.S. Cosmic Visions: New Ideas in Dark Matter (Fermilab, Batavia, 2017), Vol. 7.

[6] J. A. Dror, G. Elor, and R. Mcgehee, Directly Detecting Signals from Absorption of Fermionic Dark Matter, Phys. Rev. Lett. 124, 181301 (2020).

[7] J. A. Dror, G. Elor, and R. Mcgehee, Absorption of fermionic dark matter by nuclear targets, J. High Energy Phys. 02 (2020) 134.

[8] D. N. Spergel and P. J. Steinhardt, Observational Evidence for Selfinteracting Cold Dark Matter, Phys. Rev. Lett. 84, 3760 (2000).

[9] M. Boylan-Kolchin, J. S. Bullock, and M. Kaplinghat, Too big to fail? The puzzling darkness of massive Milky Way subhaloes, Mon. Not. R. Astron. Soc. 415, L40 (2011).

[10] K. A. Oman et al., The unexpected diversity of dwarf galaxy rotation curves, Mon. Not. R. Astron. Soc. 452, 3650 (2015).

[11] J. McDonald, Thermally Generated Gauge Singlet Scalars as Selfinteracting Dark Matter, Phys. Rev. Lett. 88, 091304 (2002).

[12] L. J. Hall, K. Jedamzik, J. March-Russell, and S. M. West, Freeze-in production of FIMP dark matter, J. High Energy Phys. 03 (2010) 080.

[13] N. Bernal, M. Heikinheimo, T. Tenkanen, K. Tuominen, and V. Vaskonen, The dawn of FIMP dark matter: A review of models and constraints, Int. J. Mod. Phys. A 32, 1730023 (2017).

[14] T. Hambye, M. H. G. Tytgat, J. Vandecasteele, and L. Vanderheyden, Dark matter direct detection is testing freeze-in, Phys. Rev. D 98, 075017 (2018).

[15] R. Essig, T. Volansky, and T.-T. Yu, New constraints and prospects for sub-GeV dark matter scattering off electrons in xenon, Phys. Rev. D 96, 043017 (2017).

[16] G. Arcadi and L. Covi, Minimal decaying dark matter and the LHC, J. Cosmol. Astropart. Phys. 08 (2013) 005.

[17] S. B. Roland, B. Shakya, and J. D. Wells, PeV neutrinos and a $3.5 \mathrm{keV}$ x-ray line from a PeV-scale supersymmetric neutrino sector, Phys. Rev. D 92, 095018 (2015).

[18] A. Fradette, M. Pospelov, J. Pradler, and A. Ritz, Cosmological constraints on very dark photons, Phys. Rev. D 90, 035022 (2014).

[19] N. Bernal, X. Chu, C. Garcia-Cely, T. Hambye, and B. Zaldivar, Production regimes for self-interacting dark matter, J. Cosmol. Astropart. Phys. 03 (2016) 018.

[20] J. Berger, K. Jedamzik, and D. G. E. Walker, Cosmological constraints on decoupled dark photons and dark Higgs, J. Cosmol. Astropart. Phys. 11 (2016) 032.

[21] R. T. Co, F. D’Eramo, L. J. Hall, and D. Pappadopulo, Freeze-in dark matter with displaced signatures at colliders, J. Cosmol. Astropart. Phys. 12 (2015) 024.

[22] J. A. Evans and J. Shelton, Long-lived status and displaced leptons at the LHC, J. High Energy Phys. 04 (2016) 056.

[23] A. G. Hessler, A. Ibarra, E. Molinaro, and S. Vogl, Probing the scotogenic FIMP at the LHC, J. High Energy Phys. 01 (2017) 100.

[24] A. Ghosh, T. Mondal, and B. Mukhopadhyaya, Heavy stable charged tracks as signatures of non-thermal dark matter at the LHC: A study in some non-supersymmetric scenarios, J. High Energy Phys. 12 (2017) 136.
[25] L. Calibbi, L. Lopez-Honorez, S. Lowette, and A. Mariotti, Singlet-doublet dark matter freeze-in: LHC displaced signatures versus cosmology, J. High Energy Phys. 09 (2018) 037.

[26] G. Bélanger et al., LHC-friendly minimal freeze-in models, J. High Energy Phys. 02 (2019) 186.

[27] J. Alimena et al., Searching for long-lived particles beyond the Standard Model at the large hadron collider, arXiv: 1903.04497.

[28] D. Curtin et al., Long-lived particles at the energy frontier: The MATHUSLA physics case, Rep. Prog. Phys. 82, 116201 (2019).

[29] A. Ariga et al. (FASER Collaboration), FASER?s physics reach for long-lived particles, Phys. Rev. D 99, 095011 (2019).

[30] J. M. No, P. Tunney, and B. Zaldivar, Probing Dark Matter freeze-in with long-lived particle signatures: MATHUSLA, HL-LHC and FCC-hh, J. High Energy Phys. 03 (2020) 022.

[31] S. Heeba and F. Kahlhoefer, Probing the freeze-in mechanism in dark matter models with $U(1)^{\prime}$ gauge extensions, Phys. Rev. D 101, 035043 (2020).

[32] C. Boehm and P. Fayet, Scalar dark matter candidates, Nucl. Phys. B683, 219 (2004).

[33] S. Knapen, T. Lin, and K. M. Zurek, Light dark matter: Models and constraints, Phys. Rev. D 96, 115021 (2017).

[34] M. R. Buckley and P. J. Fox, Dark matter self-interactions and light force carriers, Phys. Rev. D 81, 083522 (2010).

[35] M. Kaplinghat, S. Tulin, and H.-B. Yu, Dark Matter Halos as Particle Colliders: Unified Solution to Small-Scale Structure Puzzles from Dwarfs to Clusters, Phys. Rev. Lett. 116, 041302 (2016).

[36] Y. G. Kim, K. Y. Lee, and S. Shin, Singlet fermionic dark matter, J. High Energy Phys. 05 (2008) 100.

[37] L. Lopez-Honorez, T. Schwetz, and J. Zupan, Higgs portal, fermionic dark matter, and a Standard Model like Higgs at 125 GeV, Phys. Lett. B 716, 179 (2012).

[38] G. Krnjaic, Probing light thermal dark-matter with a Higgs portal mediator, Phys. Rev. D 94, 073009 (2016).

[39] T. Hambye, M. H. G. Tytgat, J. Vandecasteele, and L. Vanderheyden, Dark matter from dark photons: A taxonomy of dark matter production, Phys. Rev. D 100, 095018 (2019).

[40] N. Bernal, A. Donini, M. G. Folgado, and N. Rius, KaluzaKlein FIMP dark matter in warped extra-dimensions, arXiv: 2004.14403.

[41] C. Dvorkin, T. Lin, and K. Schutz, Making dark matter out of light: Freeze-in from plasma effects, Phys. Rev. D 99, 115009 (2019).

[42] G. Bélanger, F. Boudjema, A. Goudelis, A. Pukhov, and B. Zaldivar, micrOMEGAs5.0: Freeze-in, Comput. Phys. Commun. 231, 173 (2018).

[43] B. Batell, A. Freitas, A. Ismail, and D. Mckeen, Probing light dark matter with a hadrophilic scalar mediator, Phys. Rev. D 100, 095020 (2019).

[44] B. Batell, A. Freitas, A. Ismail, and D. Mckeen, Flavorspecific scalar mediators, Phys. Rev. D 98, 055026 (2018).

[45] D. Egana-Ugrinovic, S. Homiller, and P. Meade, Aligned and Spontaneous Flavor Violation, Phys. Rev. Lett. 123, 031802 (2019). 
[46] A. Manohar and H. Georgi, Chiral quarks and the nonrelativistic quark model, Nucl. Phys. B234, 189 (1984).

[47] X. Chu, T. Hambye, and M. H. Tytgat, The four basic ways of creating dark matter through a portal, J. Cosmol. Astropart. Phys. 05 (2012) 034.

[48] M. A. Shifman, A. I. Vainshtein, and V. I. Zakharov, Remarks on Higgs boson interactions with nucleons, Phys. Lett. 78B, 443 (1978).

[49] T. Bhattacharya, V. Cirigliano, S. Cohen, R. Gupta, H.-W. Lin, and B. Yoon, Axial, scalar and tensor charges of the nucleon from $2+1+1$-flavor lattice QCD, Phys. Rev. D 94, 054508 (2016).

[50] J. F. Donoghue, J. Gasser, and H. Leutwyler, The decay of a light Higgs boson, Nucl. Phys. B343, 341 (1990).

[51] J. Bijnens, G. Colangelo, and P. Talavera, The vector and scalar form-factors of the pion to two loops, J. High Energy Phys. 05 (1998) 014.

[52] M. Hufnagel, K. Schmidt-Hoberg, and S. Wild, BBN constraints on $\mathrm{MeV}$-scale dark sectors. Part II. Electromagnetic decays, J. Cosmol. Astropart. Phys. 11 (2018) 032.

[53] M. L. Bellac, Thermal Field Theory, Cambridge Monographs on Mathematical Physics (Cambridge University Press, 2010), https://doi.org/10.1017/CBO9780511721700.

[54] J. I. Kapusta, Finite Temperature Field Theory, Vol. 360 of Cambridge Monographs on Mathematical Physics (Cambridge University Press, Cambridge, England, 1989).

[55] N. Su, A gauge-invariant reorganization of thermal gauge theory, other thesis, 2011.

[56] E. Braaten and R. D. Pisarski, Soft amplitudes in hot gauge theories: A general analysis, Nucl. Phys. B337, 569 (1990).

[57] V. Tsytovich, Spatial dispersion in a relativistic plasma, Sov. Phys. JETP 13, 1249 (1961).

[58] H. A. Weldon, Covariant calculations at finite temperature: The relativistic plasma, Phys. Rev. D 26, 1394 (1982).

[59] H. Weldon, Dynamical holes in the quark-gluon plasma, Phys. Rev. D 40, 2410 (1989).

[60] G. F. Giudice, A. Notari, M. Raidal, A. Riotto, and A. Strumia, Towards a complete theory of thermal leptogenesis in the SM and MSSM, Nucl. Phys. B685, 89 (2004).

[61] H. A. Weldon, Effective fermion masses of order gT in high temperature gauge theories with exact chiral invariance, Phys. Rev. D 26, 2789 (1982).

[62] N. Aghanim et al. (Planck Collaboration), Planck 2018 results. VI. Cosmological parameters, arXiv:1807.06209.

[63] E. Aprile et al. (XENON Collaboration), Dark Matter Search Results from a One Ton-Year Exposure of XENON1T, Phys. Rev. Lett. 121, 111302 (2018).

[64] J. D. Lewin and P.F. Smith, Review of mathematics, numerical factors, and corrections for dark matter experiments based on elastic nuclear recoil, Technical Report No. RAL-TR-95-024, RAL, Chilton, 1996, http://cds.cern .ch/record/298578.

[65] G. Belanger, A. Mjallal, and A. Pukhov, Recasting direct detection limits within micrOMEGAs and implication for non-standard dark matter scenarios, arXiv:2003.08621.
[66] P. Agnes et al. (DarkSide Collaboration), Low-Mass Dark Matter Search with the DarkSide-50 Experiment, Phys. Rev. Lett. 121, 081307 (2018).

[67] E. Aprile et al. (XENON Collaboration), Light Dark Matter Search with Ionization Signals in XENON1T, Phys. Rev. Lett. 123, 251801 (2019).

[68] E. Aprile et al. (XENON Collaboration), Search for Light Dark Matter Interactions Enhanced by the Migdal Effect or Bremsstrahlung in XENON1T, Phys. Rev. Lett. 123, 241803 (2019).

[69] R. Agnese et al. (SuperCDMS Collaboration), Projected sensitivity of the SuperCDMS SNOLAB experiment, Phys. Rev. D 95, 082002 (2017).

[70] C. Frugiuele, E. Fuchs, G. Perez, and M. Schlaffer, Constraining new physics models with isotope shift spectroscopy, Phys. Rev. D 96, 015011 (2017).

[71] H. Leeb and J. Schmiedmayer, Constraint on Hypothetical Light Interacting Bosons from Low-Energy Neutron Experiments, Phys. Rev. Lett. 68, 1472 (1992).

[72] R. S. Willey and H.L. Yu, Neutral Higgs boson from decays of heavy flavored mesons, Phys. Rev. D 26, 3086 (1982).

[73] J. P. Lees et al. (BABAR Collaboration), Search for $B \rightarrow$ $K^{(*)} \nu \bar{\nu}$ and invisible quarkonium decays, Phys. Rev. D 87, 112005 (2013).

[74] A. V. Artamonov et al. (E949 Collaboration), New Measurement of the $K^{+} \rightarrow \pi^{+} \nu \bar{\nu}$ Branching Ratio, Phys. Rev. Lett. 101, 191802 (2008).

[75] S. Martellotti (NA62 Collaboration), $K^{+} \rightarrow \pi^{+} \nu \bar{\nu}$ decay and NP searches at NA62, Nuovo Cimento C 41, 146 (2019).

[76] F. Bergsma et al. (CHARM Collaboration), Search for axion like particle production in $400-\mathrm{GeV}$ proton-copper interactions, Phys. Lett. 157B, 458 (1985).

[77] G. G. Raffelt, Stars as laboratories for fundamental physics, 1996, https://wwwth.mpp.mpg.de/members/raffelt/ mypapers/199613.pdf.

[78] A. Burrows, M. T. Ressell, and M. S. Turner, Axions and sn 1987a: Axion trapping, Phys. Rev. D 42, 3297 (1990).

[79] P. Carenza, T. Fischer, M. Giannotti, G. Guo, G. MartínezPinedo, and A. Mirizzi, Improved axion emissivity from a supernova via nucleon-nucleon bremsstrahlung, J. Cosmol. Astropart. Phys. 10 (2019) 016.

[80] N. Ishizuka and M. Yoshimura, Axion and dilaton emissivity from nascent neutron stars, Prog. Theor. Phys. 84, 233 (1990).

[81] A. Arbey, J. Auffinger, K. Hickerson, and E. Jenssen, AlterBBN v2: A public code for calculating big-bang nucleosynthesis constraints in alternative cosmologies, Comput. Phys. Commun. 248, 106982 (2020).

[82] G. Belanger, F. Boudjema, A. Pukhov, and A. Semenov, Dark matter direct detection rate in a generic model with micrOMEGAs 2.2, Comput. Phys. Commun. 180, 747 (2009). 